\title{
Phase Noise Analysis for the Drive Loop of Capacitive MEMS Gyroscopes
}

\author{
Li Qiang $^{1, a}$, Wang Guanshi ${ }^{2}$ and Liu Xiaowei ${ }^{1,3}$ \\ ${ }^{I}$ MEMS Center, Harbin Institute of Technology, Harbin, Heilongjiang, China \\ ${ }^{2}$ China Academy of Electronics and Information Technology \\ ${ }^{3}$ Key Laboratory of Micro-Systems and Micro-Structures Manufacturing, Ministry \\ of Education, China \\ alq2518409@sina.com
}

\begin{abstract}
Phase noise is one of the most important factors in bias stability, scale factor and nonlinear, therefore, the design of gyroscope-driven circuit must consider the impact of phase noise. In this paper, a phase noise model of multi-modal noise source in drive mode of micro-gyroscope is built. It also gives the method to extract coefficients of phase noise and improves the phase noise model.
\end{abstract}

Keywords: capacitive MEMS gyroscopes; phase noise; self-excited model

\section{Introduction}

Compared with traditional gyroscope, micro-machined gyroscope based on MEMS and CMOS technology has many advantages, such as low price, small size, low power and high reliability. It is not only widely used in civil fields such as automotive electronics, medical equipment and mechanical movement, but also adopted in military areas like tactical missiles, MAV and so on. As the adoption area expands, capacitive micromachined gyroscope faces higher requirements.

The ASIC chip in capacitive micro-machined gyroscope has some problems to solve. The phase noise has bad effect on the driving stability, while analysis and optimization technique of multiple noise sources are lacked in recent research. To solve the problems, this paper has conducted a depth study on model of multiple phase noise sources of capacitive micro-machined gyroscope.

Based on the impact of multiple noise sources, a phase noise coupling model for selfexcited driving circuit with multiple noise sources is built. From this model, it can be known that the main sources include low-frequency noise, noise near the resonant frequency and noise near the multiple harmonic. After above analysis, optimized design of charge amplifier which can reduce the phase noise in driving circuit in driving mode is completed.

\section{Phase Noise Analysis for the Drive Loop}

The resonance loop model of micro-gyroscope system can be approximated to the structure shown in Figure 1, [1]. Since active electrostatic force feedback of selfexcitation circuit is equivalent to negative parallel conductance, when the micro-gyro system is stability in frequency and amplitude, the value of its negative conductance is equivalent to the value of the positive conductance of the damping system, the entire system become stability. Iinout $(t)$ can be equal to a interference signal of the self-excited resonant loop in micro-gyroscope, at time t0 a current pulse is generated from the system, which the charge of it is $\Delta \mathrm{q}$, the effect is produced as follows: 


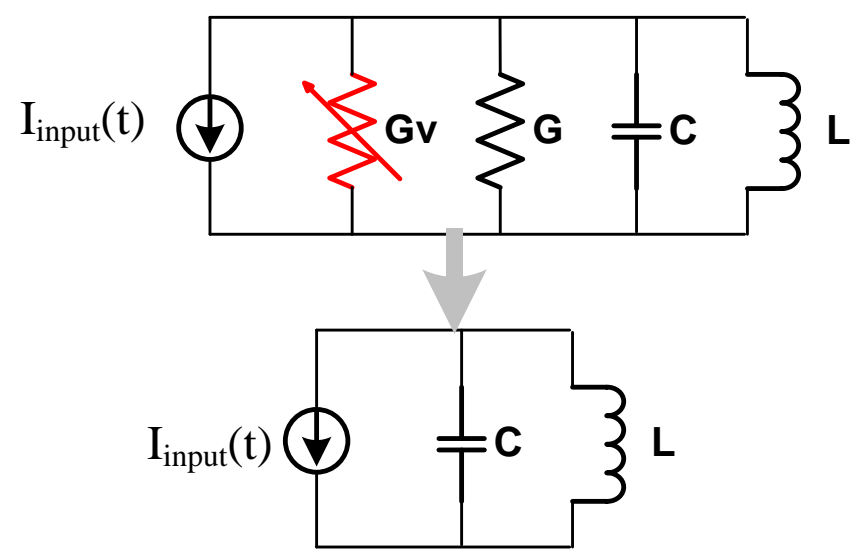

Figure 1. Simplification of Self-Excited Micro-Gyroscope Model

$U_{C}=A \cos \omega t_{0}+\frac{\Delta q}{C}$

$I_{L}=A \sqrt{\frac{C}{L}} \sin \omega t_{0}$

Where Uc is the voltage of the capacitor, IL is the inductor current value, $\mathrm{C}$ is the capacitance and $\mathrm{L}$ is inductance value, $\mathrm{A}$ is the voltage across the capacitor at resonance, $\mathrm{w}$ is the resonant frequency.

At $\mathrm{t}_{0}$, the phase is change.

$U_{C}=(A+\Delta A) \cos (\omega t+\Phi)$

$I_{L}=(A+\Delta A) \sqrt{\frac{C}{L}} \sin (\omega t+\Phi)$

At time $\mathrm{t}_{0}$, the equation is:

$U_{C}=(A+\Delta A) \cos \left(\omega t_{0}+\Phi\right)=A \cos \omega t_{0}+\frac{\Delta q}{C}$

$I_{L}=(A+\Delta A) \sqrt{\frac{C}{L}} \sin \left(\omega t_{0}+\Phi\right)=A \sqrt{\frac{C}{L}} \sin \omega t_{0}$

Because $\Phi$ is very small, which can be approximated that $\sin \Phi \approx \Phi$. Final change of phase can be obtained as follows:

$\Phi=\frac{\Delta q}{A \cdot C} \sin \omega t_{0}$

By the Formula (7), when the gyroscope system is approximately equivalent to LC oscillation loop, the phase change it related to the charge injection time.

For the resonance system of gyroscopes is not an ideal model of LC oscillator circuit, because the negative equivalent conductance changes with charge injection due to the amplitude control circuit. According to the cyclical nature of the micro-gyroscope drive signal, impulse impact response function of the phase in drive loop can be expressed as: 
$\Gamma\left(\omega_{0} \tau\right)=c_{0}+\sum_{n=1}^{\infty} c_{n} \cos \left(n \omega_{0} \tau+\theta_{n}\right)$

In Formula (8) $c_{0}, c_{n}$ are coefficients of the impulse response function, $\square_{0}$ is the resonant frequency of the micro-gyroscope, $\square$ is a time variable.

$\Phi(t)=\int_{-\infty}^{t} \frac{\Gamma\left(\omega_{0} \tau\right)}{q_{\max }} i(\tau) d \tau$

$\Phi(t)=c_{0} \int_{-\infty}^{t} i(\tau) d \tau+\sum_{n=1}^{\infty} c_{n} \int_{-\infty}^{t} i(\tau) \cos \left(n \omega_{0} \tau+\theta_{n}\right) d \tau$

When a current pulse $\mathrm{i}(\mathrm{t})$ is introduced into the system,

$i(t)=I_{n} \cos \left(n \omega_{0}+\Delta \omega\right) t$

The system phase is:

$\Phi(t)=I_{n} c_{n} \int_{-\infty}^{t} \cos \left(n \omega_{0}+\Delta \omega \tau\right) \Gamma(\tau) d \tau \approx \frac{I_{n} c_{n} \sin (\Delta \omega \tau)}{\Delta \omega}$

The phase changes under the effect of low frequency current injection $\mathrm{I}_{0} \cos \square \square \mathrm{t}$ by $\mathrm{c} 0$ can generate a low frequency component:

$\varphi(t)=\frac{I_{0} c_{0}}{C_{d} V_{d 0}} \int_{-\infty}^{t} \cos (\Delta \omega \tau) d \tau=\frac{I_{0} c_{0} \sin \Delta \omega t}{C_{d} V_{d 0} \Delta \omega}$

The impact of low-frequency interference on the phase in drive circuit is shown in Figure 2.
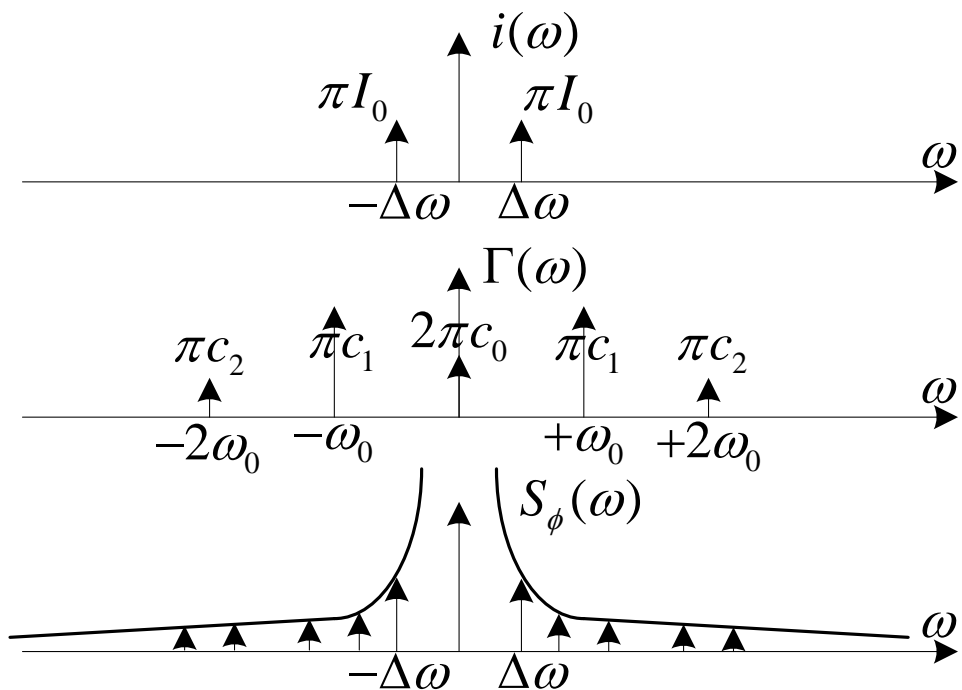

Figure 2. The Impact of Low-Frequency Interference on the Phase of MicroGyroscope Driving Signal

The phase changes under the effect of $\mathrm{I}_{\mathrm{n}} \cos \left(\mathrm{n} \square_{\mathrm{d}}+\square \square\right) \mathrm{t}$ at multiple harmonic frequency is shown as follows: 


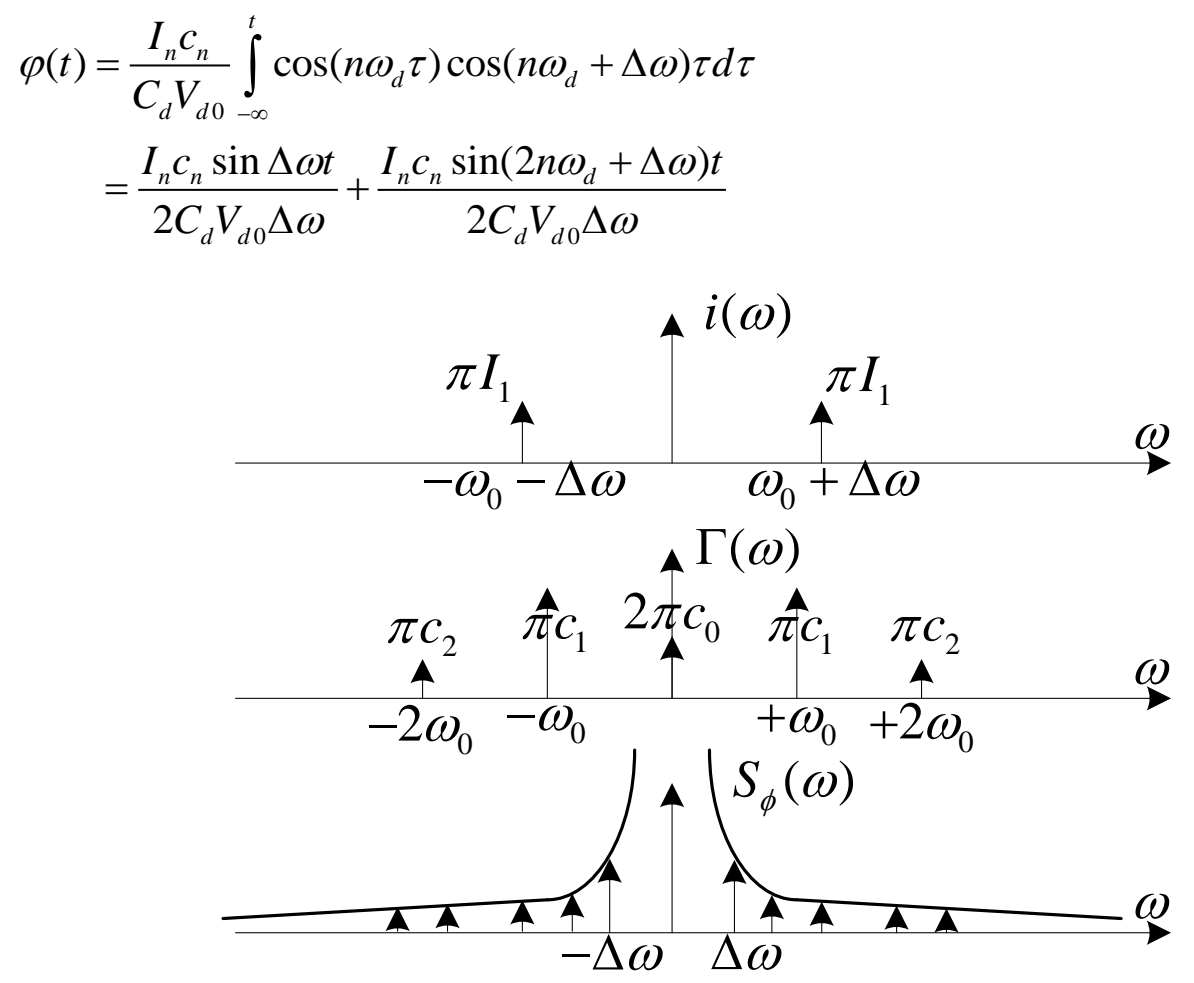

\section{Figure 3. The Impact of Multiple Harmonic Interference on the Phase of Micro-Gyroscope Driving Signal}

The Formula (13), and (14), shows that both the low-frequency or high-frequency current injection will cause the original stable phase of drive signal changes. When the phase changes by the injection current is contained, the drive frequency excitation signal can be expressed as:

$V_{d 0} \cos \left[\omega_{d} t+\varphi(t)\right]=V_{d 0} \cos \omega_{d} t \cos [\varphi(t)]-V_{d 0} \sin \omega_{d} t \sin [\varphi(t)]$

It can be simplifies to:

$V_{d 0} \cos \left[\omega_{d} t+\varphi(t)\right] \approx V_{d 0} \cos \omega_{d} t-V_{d 0} \varphi(t) \sin \omega_{d} t$

By Formula (16), it can be shown that the injected noise current of the stable driving loop will not only affect the phase of the system, but also affect the voltage of the drive signal.

\section{The Method to Extract Coefficients of Phase Noise}

According to the previous analysis, the variation of driving mode phase is relevant to coefficient $c_{0}$ and $c_{1}$, since the amplitude of the other $c_{n}$ are smaller, which made less influence on the phase of driving mode. Since the self-exciting drive loop of micromechanical gyroscope is a non-linear automatic gain control loop, the obtaining of accurate phase response function of it is very difficult, which means that it is very difficult to obtain the value of $c_{n}$, we use the following method to solve the problem' When the system is introduced a current pulse $\mathrm{i}(\mathrm{t})$ :

$i(t)=I_{n} \cos \left(n \omega_{0}+\Delta \omega\right) t$

System phase changes: 
$\Phi(t)=I_{n} c_{n} \int_{-\infty}^{t} \cos \left(n \omega_{0}+\Delta \omega \tau\right) \Gamma(\tau) d \tau \approx \frac{I_{n} c_{n} \sin (\Delta \omega t)}{\Delta \omega}$

The change of resonance signal caused by phase change is:

$\cos \left(\omega_{0} t+\Phi(t)\right)=\cos \omega_{0} t \cdot \cos \Phi(t)-\sin \omega_{0} t \cdot \sin \Phi(t) \approx \cos \omega_{0} t-\sin \omega_{0} t \cdot \Phi(t)$

Formula (18), (19) shows that:

$\cos \left(\omega_{0} t+\Phi(t)\right)=\cos \omega_{0} t+\frac{I_{n} c_{n}}{2 \Delta \omega}\left(\cos \left(\omega_{0} t+\Delta \omega t\right)-\cos \left(\omega_{0} t-\Delta \omega t\right)\right)$

It can be seen that if there is a voltage or current injection at this time, the magnitude of the system will change, Figure 4, shows phase response of the system when the voltages of injected pulse signal in the system are $20 \mu \mathrm{V}, 40 \mu \mathrm{V}, 60 \mu \mathrm{V}, 80 \mu \mathrm{V}, 100 \mu \mathrm{V}$. It can be seen from Figure 4, the system phase increases with the amplitude of injection voltage.

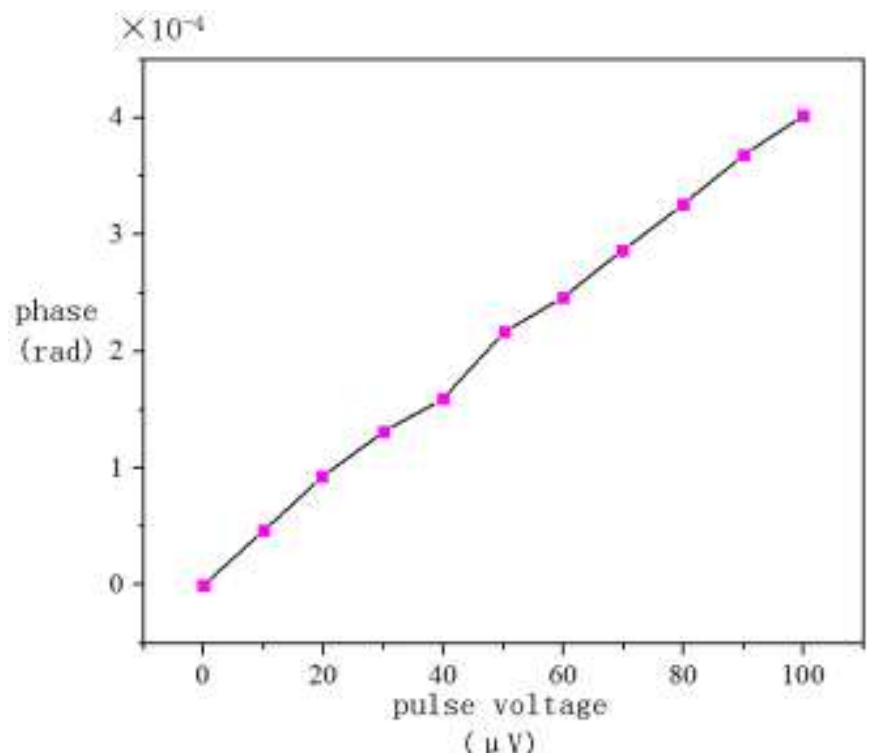

Figure 4. The Relationship between Pulse Ampitude and Phase

According to the above test results and formulas (20), when resonance system is injected using a fixed-frequency $\mathrm{n} \square_{0}+\square \square$ sinusoidal voltage or current signal, the amplitude of coefficient $c_{n}$ can be learned by observation by frequency ( $\square \square \square \square$ ) or $(\square \square \square \square)$. In this paper, by applying a voltage pulse to the input of the automatic gain control unit, and then testing the results of specific phase response, the corresponding coefficients can be obtained. 


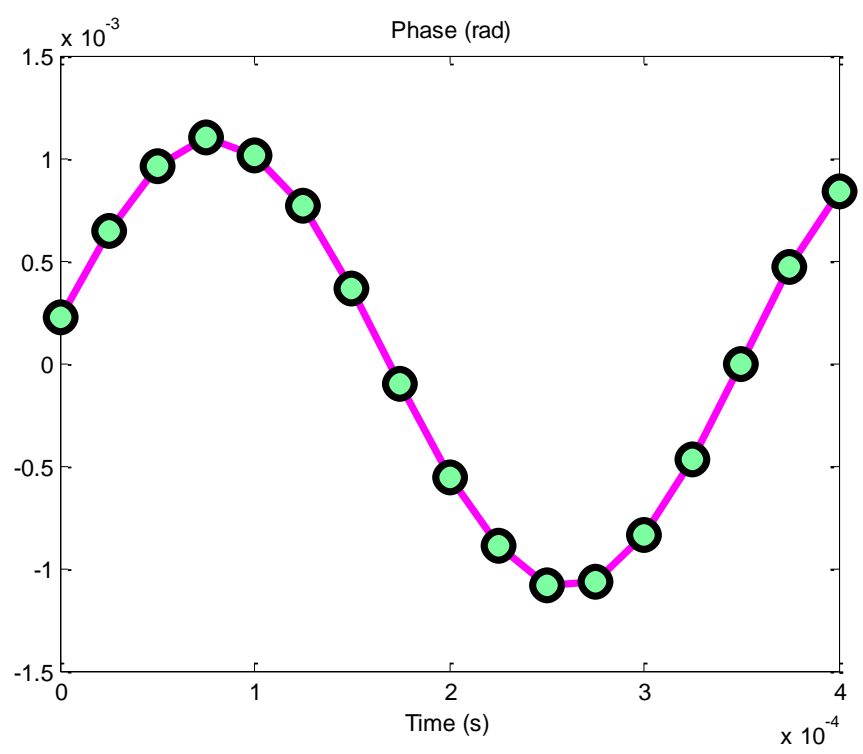

Figure 5. Micromechanical Gyroscope Self-Excitation Loop Phase Response
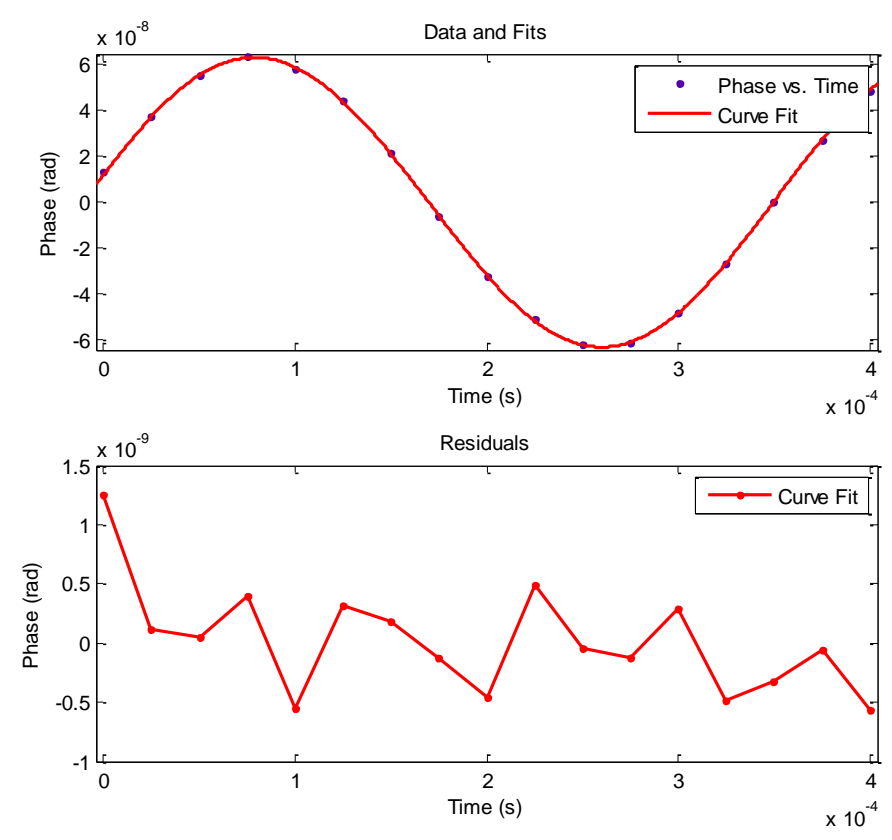

Figure 6. Phase Response Fitting Curve

The function of fitting curve is:

$\Gamma(t)=a_{0}+b_{1} \sin \left(\omega_{x} t\right)+b_{2} \cos \left(\omega_{x} t\right)+c_{1} \sin \left(2 \omega_{x} t\right)+c_{2} \cos \left(2 \omega_{x} t\right)$

The coefficient is shown in Table 1.

Table 1. Fitting Equation Coefficients

\begin{tabular}{c|c|c|c|c}
\hline$a_{0}$ & $b_{1}$ & $b_{2}$ & $c_{1}$ & $c_{2}$ \\
\hline$-3.697 \mathrm{e}-11$ & $6.1777 \mathrm{e}-8$ & $1.16 \mathrm{e}-8$ & $2.802 \mathrm{e}-10$ & $1.816 \mathrm{e}-10$ \\
\hline
\end{tabular}


Dynamic analyzer HP35670A and oscilloscope TDS2012B are used in the test. Electrostatic modulation drive signal of micro-gyroscope is shown in Figure 4-21, highfrequency square wave is used in modulation of mass velocity signal, and then the modulated driving voltage signal is applied to the Micro-gyroscope drive comb.

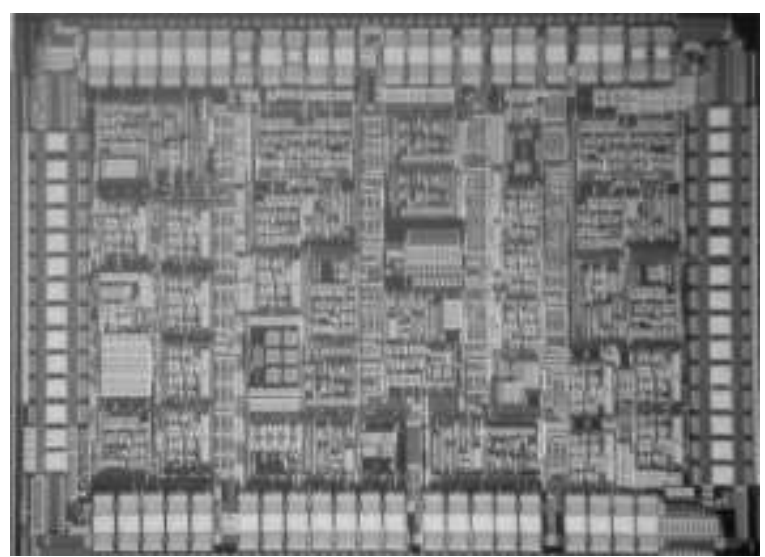

Figure 7. Photograph of the ASIC of Micro-Gyroscope

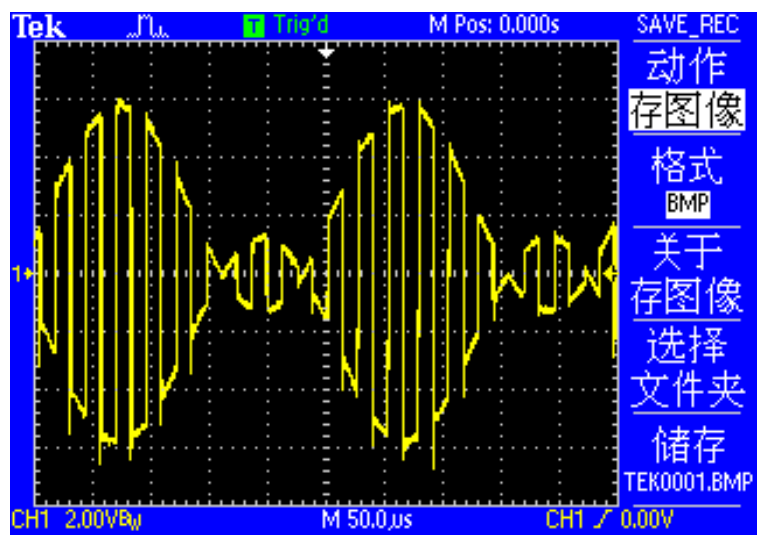

Figure 8. Electrostatic Modulation Drive Signal of Micro-Gyroscope

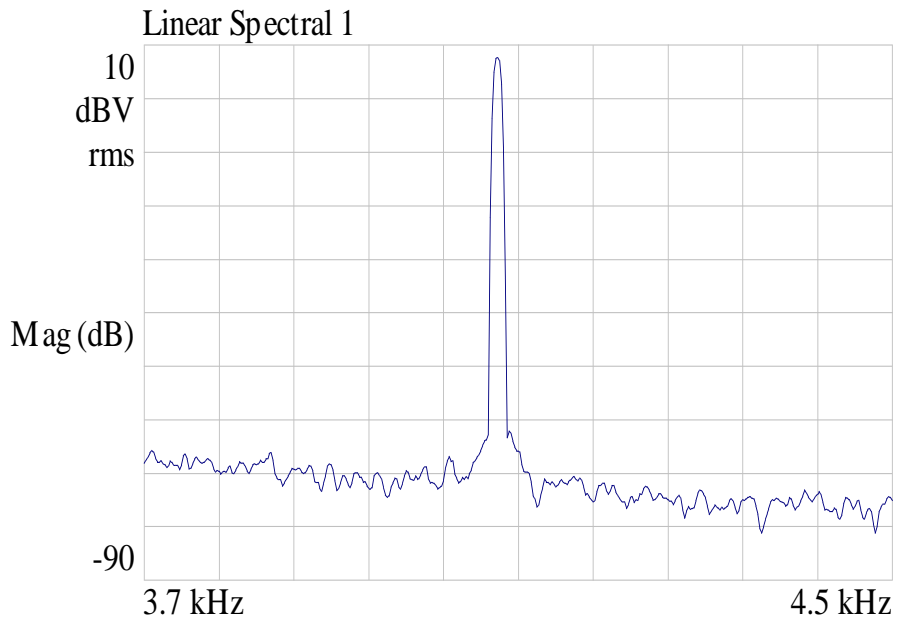

Figure 9. Test Spectrum of Closed-Loop Self-Excited Drive Motion 


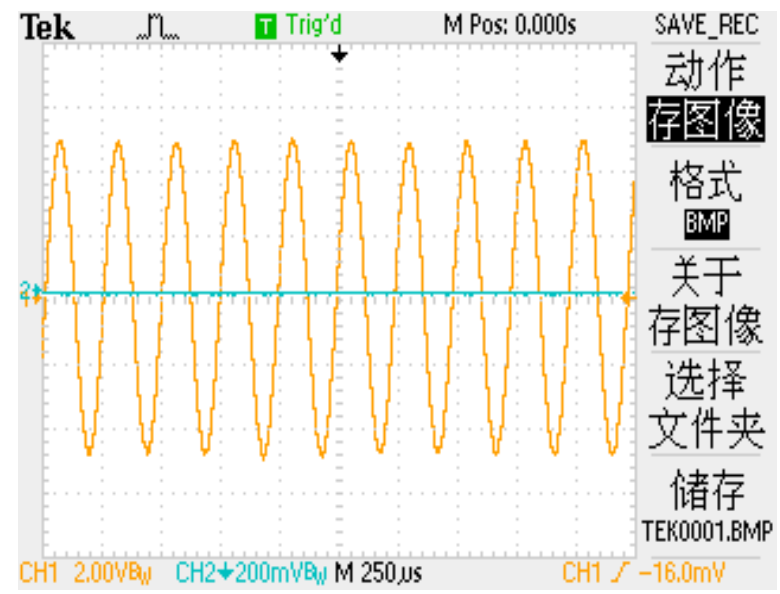

\section{Figure 10. Time-Domain Test Result of Closed-Loop Self-Excited Drive Motion}

\section{Summary}

In this paper, a phase noise model of multi-modal noise source in drive mode of microgyroscope is built, the influencing factors of the phase noise of driving oscillation loop are low frequency near DC glitches and multiple harmonic of drive resonant frequency. By quantitative analysis of the phase noise at related frequency of the charge amplifier and noise injection experiments, it can be optimized that the phase noise of driving loop is impact greater by low-frequency noise and the noise at resonant frequency. It also gives the method to extract coefficients of phase noise and improves the phase noise model.

\section{References}

[1] S. Billat, H. Glosch and M. Kunze, "Micromachined Inclinometer with High Sensitivity and Very Good Stability", Sensors and Actuators A, vol. 97, no. 1, (2002), pp. 125-130.

[2] S. Mikko, A. Lasse and K. Halonen, "Effects of Synchronous Demodulation in Vibratory MEMS Gyroscopes:A Theoretical Study", IEEE Sensors Journal, vol. 8, no. 10, (2008), pp. 1722-1733.

[3] S. Sangkyung, T. S. Woon and K. Changjoo, "On the Mode-Matched Control of MEMS Vibratory Gyroscope via Phase-Domain Analysis and Design”, IEEEASME Transactions on Mechatronics, vol. 14, no. 4, (2009), pp. 446-455.

[4] A. Hajimiri and T. H. Lee, "The Design of Low Noise Oscillators", America, Kluwer Academic Pulbishers, (1999), pp. 231-246.

[5] R. P. Leland, "Mechanical Thermal Noise in MEMS Gyroscopes", IEEE Sensors Journal, vol. 5, no. 2, (2005), pp. 493-500.

[6] A. K. Konstantin and G. M. Robert, "Phase Noise in LC Oscillators", IEEE Journal of Solid-State Circuits, vol. 35, no. 8, (2000), pp. 1244-1248.

[7] K. M. Sebastian and Z. Stefan, "CMOS LC-Oscillator Phase-Noise Analysis Using Nonlinear Models", IEEE Transactions on Circuits and Systems-I: Regular papers, vol. 51, no. 4, (2004), pp. 664-677.

[8] M. Jayanta, R. Patrick and A. Siraj, "An Analytic Circuit-Based Model for White and Flicker Phase Noise in LC Oscillators", IEEE Transactions on Circuits and Systems-I: Regular papers, vol. 54, no. 7, (2007), pp. 1584-1598.

[9] H. Rashtian and A. R. Dehghani, "New Model for Contribution of Triode Noise to Phase Noise of CMOS LC Oscillators", IEEE Transactions on Circuits and Systems-I: Regular papers, vol. 32, no. 2, (2009), pp. 1123-1132.

[10] L. He, Y. P. Xu and M. Palaniapan, "A State-Space Phase-Noise Model for Nonlinear MEMS Oscillators Employing Automatic Amplitude Control", IEEE Transactions on Circuits and Systems-I: Regular Papers, vol. 57, no. 1, (2010), pp. 189-199.

[11] O. Oliaei, "Noise Analysis of Correlated Double Sampling SC-integrators", Proc. IEEE Int. Symp. Circuits and Systems. Phoenix, AZ, USA, (2002), pp. 445-448.

[12] M. Nick and A. Mortazawi, "Oscillator Phase-Noise Reduction Using Low-Noise High-Q Active Resonators”, IMS, Anaheim, CA, United states, (2010), pp. 276-279. 
[13] P. Ward and A. Duwel, "Oscillator Phase Noise: Systematic Construction of an Analytical Model Encompassing Nonlinearity", IEEE Transactions on Ultrasonics, Ferroelectrics, and Frequency Control, vol. 58, no. 1, (2011), pp. 195-205.

[14] M. Pardo, L. Sorenson and F. Ayazi, "An Empirical Phase-Noise Model for MEMS Oscillators Operating in Nonlinear Regime”, IEEE Transactionson Circuitsand Systems, vol. 59, no. 5, (2012), pp. 979-988.

[15] W. Loh, S. Yegnanarayanan and R. Ram, "Unified Theory of Oscillator Phase Noise I: White Noise", IEEE Transactionon Microwave Theoryand Techniques, vol. 61, no. 6, (2013), pp. 2371-2381.

[16] Y. Jiao, D. Wu and D. Chen, "The Robust Stability Analysis of Secondary Uncertainty Dynamical System", Journal of Harbin University of Science and Technology, vol. 9, no. 5, (2004), pp. 97-99. 
International Journal of Signal Processing, Image Processing and Pattern Recognition Vol. 10, No. 1 (2017) 